\title{
Application of platelet rich fibrin in the treatment of ingival and bone necrosis following the use of paraformaldehyde containing devitalizing paste
}

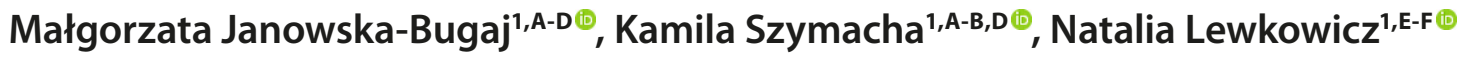 \\ ${ }^{1}$ Department of Periodontology and Oral Mucosal Diseases, Medical University, Lodz, Poland \\ A - Research concept and design, B - Collection and/or assembly of data, C - Data analysis and interpretation, \\ $D$ - Writing the article, E-Critical revision of the article, F- Final approval of article.
}

Janowska-Bugaj M, Szymacha K, Lewkowicz N. Application of platelet rich fibrin in the treatment of ingival and bone necrosis following the use of paraformaldehyde containing devitalizing paste. J Pre-Clin Clin Res. 2021; 15(2): 56-62. doi: 10.26444/jpccr/134372

\begin{abstract}
Introduction. Paraformaldehyde has been used in endodontics when effective anaesthesia could not be achieved. However, this agent has many adverse effects including gingival, bone necrosis, osteomyelitis and allergy on account of systemic exposure. Therefore, the use of pastes containing paraformaldehyde should be avoided in contemporary dental practice. Objective. The aim of this case report was to present the application of platelet-rich fibrin(PRF) in combination with a coronally advanced flap in the treatment of gingival and bone necrosis associated with paraformaldehyde containing devitalizing paste toxicity. PRF is an autogenous resorbable membrane composed of platelets, cytokines, leukocytes and growth factors.

Results and conclusions. Four weeks following burgery, the wound was completely healed with epithelial cells covering the interdental crater. On the basis of literature and of the present case, it can be stated that the use of PRF significantly reduces postoperative pain and promotes tissue healing.
\end{abstract}

\section{Key words}

bone necrosis, devitalization, paraformaldehyde, platelet rich fibrin (PRF)

\section{INTRODUCTION}

Effective pain management and performing painless root canal treatment is a major challenge for the dental practitioner. It is often difficult to obtain effective anaesthesia in mandibular molars [1,2]. According to Cohen et al. [2], after nerve block, $39 \%$ of patients that suffered from irreversible pulpitis of mandibular molars remained sensitive to the subsequent dichlorodifluoromethane cold test. Devitalizing agents were designed to solve that problem. Paraformaldehydecontaining devitalizing pastes, i.e. Devipasta (ChemaElektromet, Poland), Caustinerf Fort (Septodont, Lithuania), Depulpin (VOCO, Germany) are still used in dentistry for the treatment of irreversible pulpitis. Devipasta contains $450 \mathrm{mg}$ of paraformaldehyde and $370 \mathrm{mg}$ of lidocaine per one gram of [3]. Inclusion of the anesthetic component, lidocaine may prevent toothache caused by paraformaldehyde [3]. In the pulp chamber, paraformaldehyde and its depolymerization product formaldehyde penetrate gradually into the pulp, leading to its necrosis and mummification [3]. Devitalizing paste should remain in the tooth for a maximum period of two weeks [4]. It is crucial to use glass-ionomer material as a temporary filling to provide good sealing, and to avoid leakage of the devitalizing agent into the marginal periodontium [3].

The cytotoxic effects of paraformaldehyde-containing paste used in dentistry have been described previously $[5,6$,

Address for correspondence: Małgorzata Janowska-Bugaj, Department of Periodontology and Oral Mucosal Diseases, Medical University, Pomorska 251, 92-213 Lodz, Poland

E-mail: malgorzata.janowska@umed.lodz.pl

Received: 30.09.2020; accepted: 15.03.2021; first published: 01.04.2021
$7,8]$, and the safety of paraformaldehyde has been questioned by numerous studies $[1,4,5,6,9,10]$. Paraformaldehyde may be responsible for a range of local adverse effects, such as gingival necrosis, bone necrosis, osteomyelitis and paresthesia as a result of nerve damage $[1,7]$. Exposure to formaldehyde may also cause irritation of the eyes and upper respiratory tract, nasal congestion, pulmonary oedema, choking, dyspnea and chest tightness [1].

Type I and type IV allergic reactions to paraformaldehyde have also been reported [5]. Type I hypersensitivity reaction is a life-threatening allergic response involving massive histamine release, which can lead to a drop in blood pressur, and even sometimes to death. Type I hypersensitivity to formaldehyde showed time differences between exposure and reaction development. First symptoms usually occur 2-12 hours after the placement of the paraformaldehyde agent [5]. Formaldehyde penetrates dentinal tubules and diffuses into the periodontium, thus, $30 \mathrm{~min}$ following placement of the devitalizing paste, formaldehyde levels could be measured outside tooth tissues, and its concentration raises logarithmically for $24 \mathrm{~h}$ [11]. These findings explain why type I hypersensitivity reaction may occur several hours after dental procedure. Type IV hypersensitivity reaction usually manifests in the oral mucosa and skin with different clinical patterns. Clinical manifestation includes maculopapular or pustular exanthaema and bullous reactions (i.e. StevensJohnsons Syndrome or toxic epidermal necrolysis) which are characterized by widespread keratinocyte apoptosis [12].

Periodontal tissue damage after the use of paraformaldehydecontaining pastes has been reported in the literature $[1,2,6]$. Hülsmann et al. [13] showed that a small marginal leakage of 
a temporary filling, or a small iatrogenic perforation of the chamber floor, can lead to paste penetration to periodontal tissues causing significant damage to the periodontal ligament, bone and gingiva. Ozgöz et al. [9] emphasized that chemical substances used in endodontics affect peripheral tissues since dental pulp is united with periodontium via a net of dentinal cana, as well as via iatrogenic communication routes. Therefore, any use of devitalizing agents in dentistry should be carefully considered.

\section{OBJECTIVE}

The aim of this case report was to present the application of platelet-rich fibrin (PRF) in the treatment of gingival and bone necrosis as a complication following the use of paraformaldehyde-containing devitalizing paste.

\section{CASE REPORT}

A 33-year-old generally healthy woman presented with the chief complaint of severe localized pain of gingiva at the site of the upper left premolars, which was not relieved following Ketonal forte (Sandoz, Poland) self-administration. Aside from being a heavy smoker (more than 20 cigarettes/day), her general medical history was irrelevant to the present symptoms. She reported undergoing treatment of tooth 25 one month ago when a devitalizing agent (Devipasta, Chema-Elektromet, Poland) was placed in the mesio-occlusal cavity of tooth 25. Four days later, the patient started to feel pain in the region of dental treatment and observed that gingiva surrounding the treated tooth had become yellowish, and the temporary filling was partially missing. Six days following initial dental treatment, the patient visited another dental office because of persistent, severe pain. The dental practitioner diagnosed gingival necrosis, rebuitd the mesial wall of tooth 25 with a composite material, proceeded with endodontic treatment, and referred the patient to a periodontist.

A month after placement of devitalizing agent, the patient presented herself at the Department of Periodontology and Oral Mucosal Diseases of the Medical University in Lodz. Intra-oral examination revealed papilla in the interproximal area of teeth 24-25 and attached gingiva loss on the buccal surface of the tooth 25 . The ridge of the buccal aspect of alveolar bone crest was exposed and an interproximal alveolar bone crater was visible in the region of teeth 24-25 (Fig. 1).

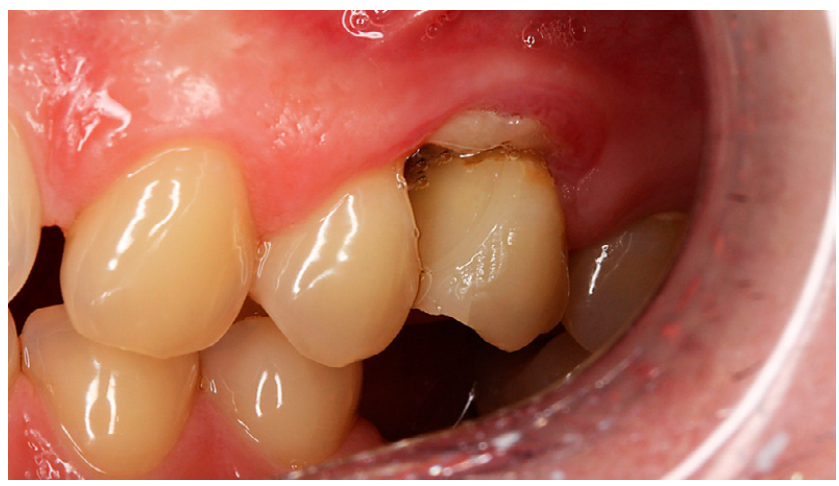

Figure 1. Gingival and bone necrosis at the site of teeth $24-25$ on the day of the first appointment

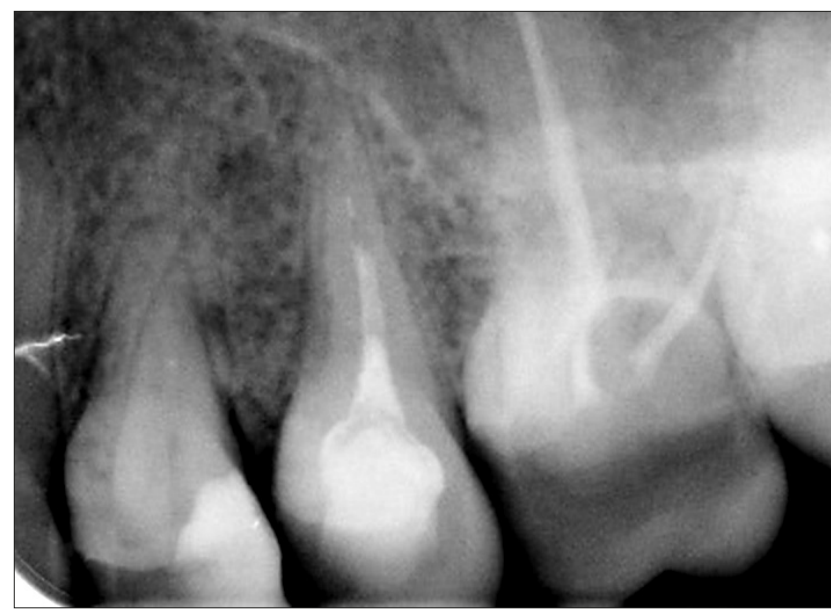

Figure 2. Periapical radiography made during the patient's first appointment

Periapical radiography showed a subtle demarcation line in the alveolar bone and a temporary filling in the root of the tooth 25 (Fig. 2). No radiolucency was detected in the apical region of the upper left premolars. Exploration of the buccal aspect of alveolar bone with a periodontal probe revealed the demarcation line (Fig. 3). The exposed bone was greyish and sensitive with no bleeding on probing. Flap surgery was scheduled for the next day, and the patient received clindamycin (Dalacin C Pfizer, Belgium) 0.3 g every six hours for six days. The patient was also advised to discontinue, or at least drastically reduce cigarette smoking. Due to the difficult-to-heal wound associated with bone sequestration in the region of the upper left premolars, PRF was planned to be applied during the flap surgical procedure.

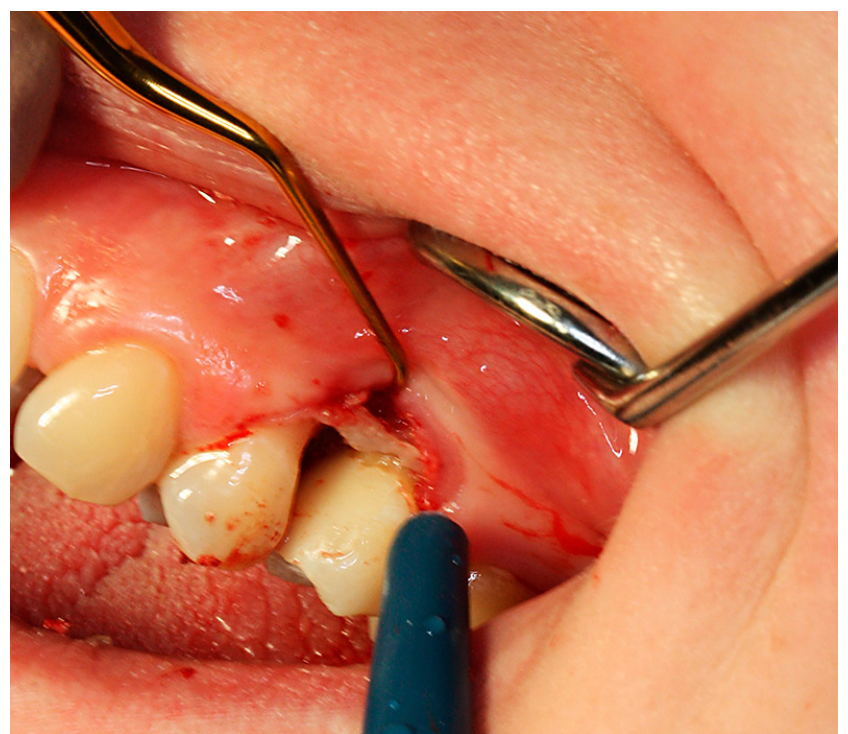

Figure 3. Necrotic buccal bone plate with demarcation line

Before the procedure, informed consent was obtained, and venous blood $\left(18 \mathrm{ml}\right.$ ) was sampled with Vacuette ${ }^{\varpi}$ (Greiner Bio One International AG, Germany) into two separate blood collecting tubes. The samples were immediately centrifuged at 2,100 rpm for 12 min using a MPW 370 centrifuge (MPW Med Instruments, Poland). This method allows blood separation into three visible layers, i.e. a cell-free layer occupying the uppermost part of the tube (PPP- plateletpoor plasma), a red blood cell (RBC) layer that occupies the 


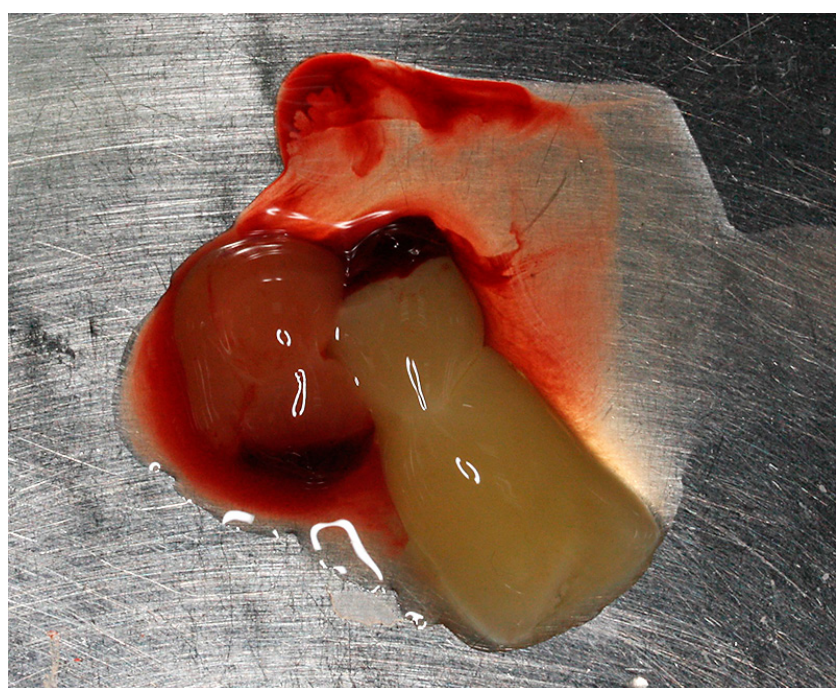

Figure 4. PRF with visible $2 \mathrm{~mm}$-border of the red layer

lowermost part of the tube, and a PRF layer located between the two [14]. The upper, yellow layer was removed. To form PRF membrane, the middle layer was cut with scissors $2 \mathrm{~mm}$ below the border with the RBC layer (Fig. 4). Before the flap surgery, the oral cavity was rinsed with $0.2 \%$ chlorhexidine solution for one minute. Local infiltrating anaesthesia was administered buccally and palatally (Citocartin 100, Molteni Dental, Italy) in the region of teeth 24-25. The mucoperiosteal trapezoid flap was raised from the buccal aspect of the teeth 24-25 and the non-bleeding grayish bone and granular tissue were removed using curettes and excavators. Bony edges were smoothed with burs in a low-speed handpiece with a saline solution irrigation. The removed bone was submitted to histopathological examination (Fig. 5). The buccal flap was mobilized by periosteal horizontal incision at the base of the flap to allow its coronal advancement. The bone crater (Fig. 6) on the buccal aspect was covered with a double PRF membrane. The buccal flap was then coronally positioned and stabilized with 6-0 monofilament polypropylene (Dafilon, B. Braun, Rubi, Barcelona, Spain) vertical mattress and single interrupted sutures (Fig. 7). The patient was provided with recommendations on the use of $0.2 \%$ chlorhexidine solution three times a day and $0.2 \%$ chlorhexidine gel twice a day. To reduce postoperative pain, ibuprofen (Nurofen forte Reckitt Benckiser, UK) 400 mg everyeight hours was recommended. Because the patient reported gastrointestinal complications, probably caused by the clindamycin, the antibiotic was switched to amoxycillin 1.0 every $12 \mathrm{~h}$. A follow-up appointment was scheduled one week later.

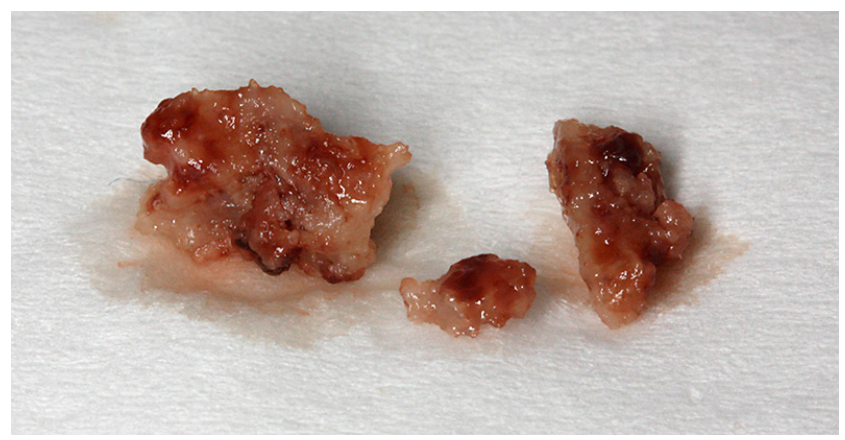

Figure 5. Excavated necrotic bone

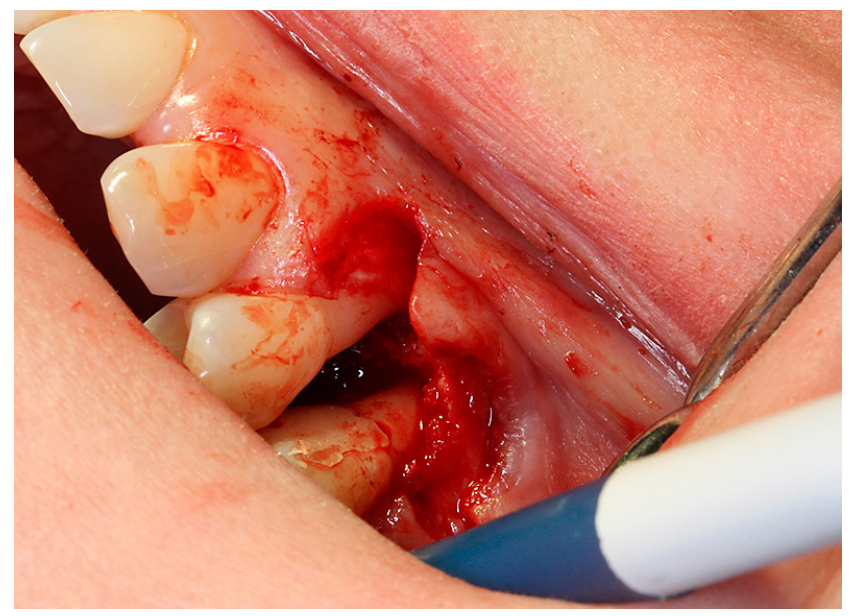

Figure 6. Crater in the interdental space after removal of necrotic bone

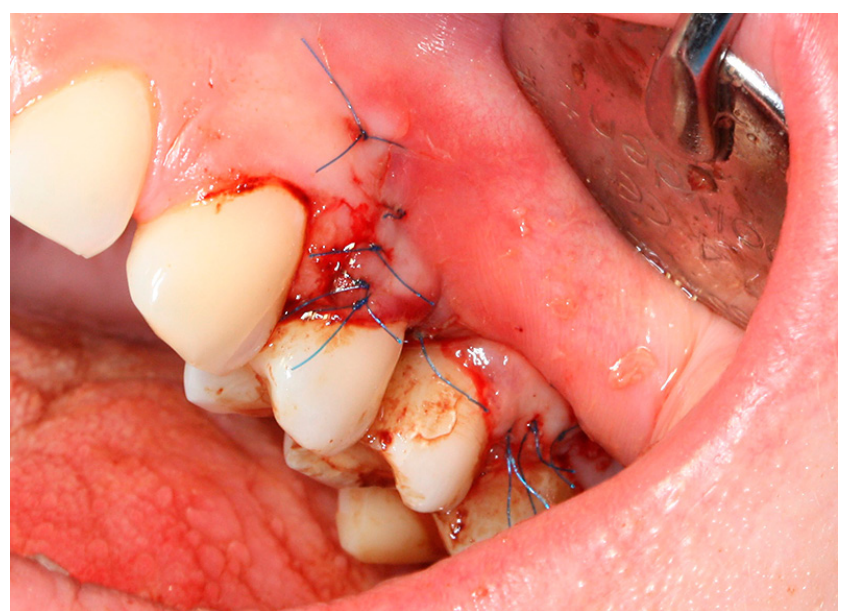

Figure 7. Sutures closing the wound. Oral vestibule became considerably shallower

Due to personal reasons, the patient came for a followup appointment two-weeks after the surgical procedure. Intra-oral examination revealed no bone exposure in the buccal and interproximal area of teeth 24-25; however, soft tissue was not completely healed (Fig. 8). Fibrin exudate and granulation tissue were still visible. The interproximal area of the 24-25 was characterized by a noticeable soft tissue and hard tissue loss in the vertical dimension. The periapical radiography showed a loss of interproximal bone

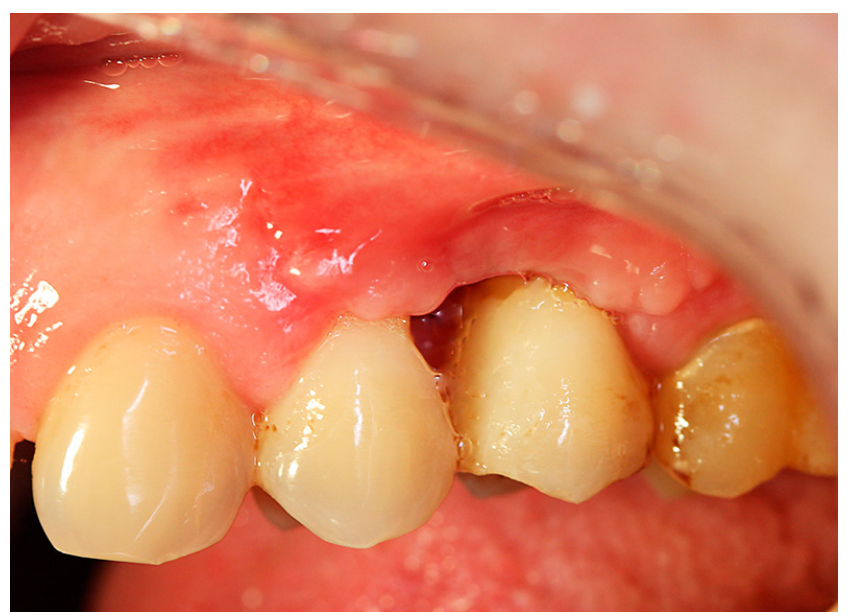

Figure 8. Clinical presentation two weeks after surgery 


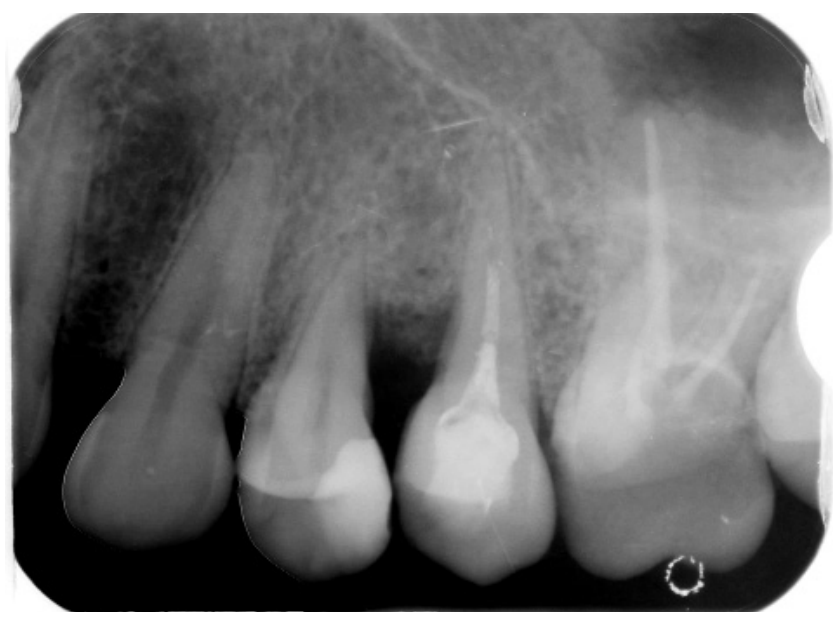

Figure 9. Periapical radiography two weeks after surgery

up to $1 / 2$ of the root length in teeth $24-25$ (Fig. 9). The result of histopathological examination correlated with the clinical finding of bone sequestration, i.e. showing normal and necrotically-changed bone lamella, early granulation tissue, and infiltration of neutrophils.

The patient stated that she took only three tablets of ibuprofen $400 \mathrm{mg}$ on the day of the surgical procedure. She also reported minor oedema of the left cheek after the procedure which resolved on the third day after the operation. One month after surgical treatment, the wound was completely healed. However, the buccal aspect of keratinized gingiva and interdental papilla were lost, exposing the cementoenamel junction in both premolars, the vestibule depth decreased, with evidence of marginal tissue pulling by the buccal frenulum attachment (Fig. 10). Due to a significant post-surgical bone loss in the interdental space of teeth 24-25, tooth 24 became hypersensitive to hot and cold. Because of the severe tissue destruction, the patient required further mucogingival surgery with tissue grafting to reconstructhe periodontal tissues. However, because of the treatment costs and unpredictable prognosis (the patient was still smoking around 10-15 cigarettes per day), the patient did not accept any further surgical treatment.

The patient did not appear at the Department of Periodontology and Oral Mucosal Diseases for the subsequent follow-up visits. She finally came three years after the surgery.

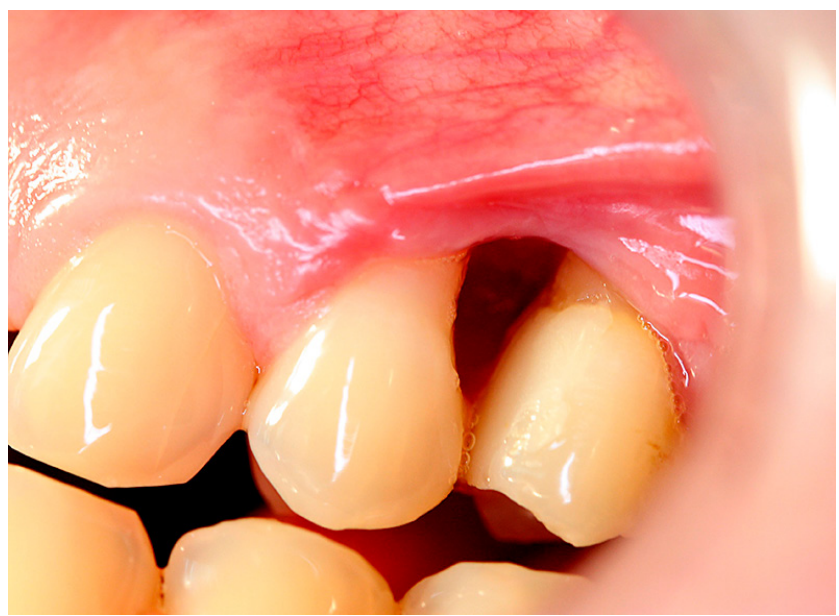

Figure 10. One month after surgery. Vestibule Has become considerably shallower Absence of the interproximal dental papilla between teeth $24-25$

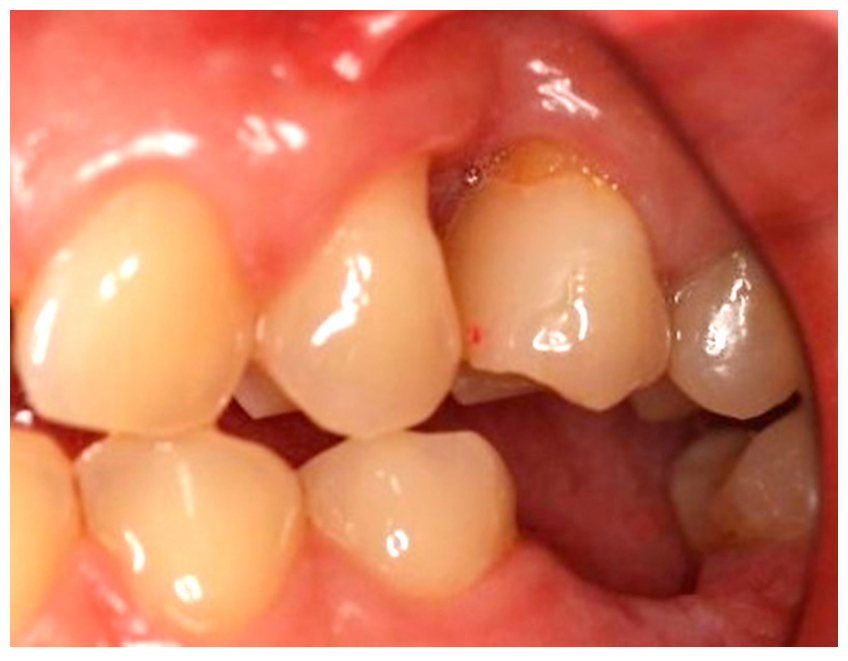

Figure 11. State of periodontal tissue three years after of surgery

The patient has not reported any discomfort in the treated area. Both upper left premolars were preserved, and tooth 24 was vital. Clinical examination showed the absence of buccal aspect of the interdental papilla between teeth 24-25 and exposure of cementoenamel junction in this area (Fig. 11), but no increased probing depth or bleeding on probing was detected. In comparison to the clinical situation two weeks postoperative, a band of attached gingiva and vestibule depth were reconstructed. The periapical radiography of the operated area showed horizontal bone loss, but the bone was well-mineralized with clearly visible lamina dura (Fig. 12). The contact point between the upper left premolars was lacking. Despite a tissue defect in the interproximal area of teeth $24-25$, the patient was satisfied with the treatment results and did not wish to undergo any further mucogingival surgery or prosthetic treatment to reconstruct the interproximal contact point in the region of the teeth.

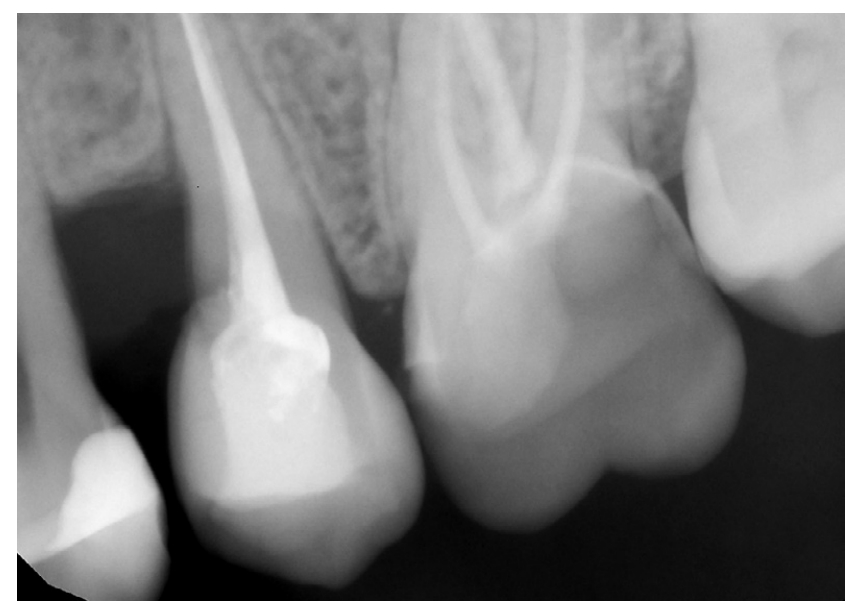

Figure 12. Periapical radiography made 3 years after surgery

\section{DISCUSSION}

In the past, effective analgesia was difficult to obtain in patients with severe toothache. Therefore, devitalizing arsenic-based or paraformaldehyde-based pastes were commonly used by dental practitioners. Nowadays, the use of devitalizing pastes is less common; therefore, patients with 
complications following the use of these agents are rarely seen in dental practice; however, every dental practitioner should know how to deal with such complications.

The case study presented the successful treatment of devitalizing paste-induced gingival and bone necrosis at 3-year follow-up using a coronally advanced flap (CAF) procedure in combination with PRF. Placement of the devitalizing agent resulted in the necrosis of gingiva and bone in the buccal and interproximal area of the treated tooth. This complication was caused by a leakage of the devitalizing paste into periodontal tissues via unsound mesio-occlusal temporary tooth filling.

Treatment of local complications after the use of paraformaldehyde-containing paste requires the removal of necrotic tissues. As a result, a considerable loss of gingiva and bone may develop [4]. Lee et al. [1] reported two clinical cases in which paraformaldehyde-containing paste was used for the treatment of irreversible pulpitis. The paste caused local osteomyelitis around the treated teeth. The treatment of choice was an extraction of the causative teeth together with the adjacent teeth, decortication and saucerization of the mandible. Surgical treatment of necrotic tissue caused by paraformaldehyde- or arsenic-containing paste, together with tooth/teeth extraction has also been reported $[4,6,15]$. On the contrary, rapid relief of pain and tooth preservation following paraformaldehyde-containing paste-induced tissue necrosis was achieved using PRF and CAF [16]. Therefore, to improve tissue healing, in this case study it was decided to combine necrotic tissue removal with PRF application, together with the CAF procedure.

PRF is a product of centrifugation of peripheral blood in the centrifuge for about $12-14 \mathrm{~min}$ at $1,300-2,700 \mathrm{rpm}$ [17], and is a 3 -dimensional structure with fibrin as its matrix. The PRF network includes platelets, white blood cells and plasma growth factors, such as platelet growth factor (PDGF), insulin growth factor (IGF), transforming $\beta$ growth factor (TGF- $\beta$ ), vascular endothelial growth factor (VEGF), epidermal growth factor (EGF), basic fibroblast growth factor (bFGF), hepatocyte growth factor (HGF), thrombospondin-1 (TSP-1), insulin-like growth factors I and II (IGF I, II), cytokines and interleukins, e.g. IL-1 $\beta$, IL-4 and IL- $6[17,18,19,20]$. These growth factors are released from PRF for 7-10 days [21, 22]. The simple procedure for obtaining PRF is based on the natural properties of blood. The blood is collected from the patient and centrifuged without any anticoagulants or artificial activators. PRF can be used as an autologous material for the regeneration of soft tissue and bone in the form of membranes or plugs [18]. PRF stimulates proliferation and differentiation of osteoblasts, endothelial cells and fibroblasts, accelerating tissue healing and regeneration. It also reduces postoperative bleeding and pain [20].

In the current case study, the bone and gingival loss following necrotic tissue removal were significant, but the treated tooth, as well as the adjacent teeth, was saved and remained stable at 3-year follow-up. Using CAF procedure and PRF, primary closure of the wound was attempted, but unfortunately the tissue defect was so big that the wound healed per secundam. Nevertheless, rapid relief of pain was demonstrated, similar to that attained by Naik et al. [16].

Before surgical removal of necrotic bone, systemic antibiotics should be administered and continued after the surgery. Commonly, amoxycillin $0.5 \mathrm{~g}$ alone or together with metronidazole 0.2 g every eight hours for seven days $[4,23,24]$, or clindamycin $0.6 \mathrm{~g}$ every $12 \mathrm{~h}$ were prescribed [15]. In the present case, initially, clindamycin $0.3 \mathrm{~g}$ was administered due to its good bone penetration. Because the patient-reported gastrointestinal complications, probably caused by the clindamycin, the antibiotic was changed to amoxycillin 1.0 g every 12 hours. Most authors advise using a rinsing solution containing $0.2 \%$ chlorhexidine $[4,6]$ and chlorhexidine gel [6], and the same was recommended in the presented case. Some authors advocate histopathology of the removed necrotic tissues, as performed in the present case study [23]. Histopathological examination allows the exclusion of any other possible diagnosis (e.g. malignancy, and confirmed clinical diagnosis in this case study.

According to the literature, wound healing after necrosis caused by paraformaldehyde-containing paste occurs from four to seven weeks after surgery $[4,6]$. In this case study, PRF was used to improve healing. When compared with other biomaterials used in dentistry, PRF has numerous advantages as an autologous material with a low risk of allergic reactions or cross- infections [25]. PRF preparation is a short-time and cost-effective procedure which makes it a reasonable alternative to resorbable and non-resorbable membranes in many clinical situations [25]. In the presented case, the wound was completely healed one month following surgery, the epithelial tissue had covered the interdental crater, and the patient did not suffer from post-operative pain. PRF is used in many fields of dentistry - oral and maxillofacial surgery, periodontics and implant surgery. Many researchehave observed improved tissue healing and reduced postoperative pain after surgical procedures combined with the use of PRF $[26,27,28]$. PRF can be also used in the socket preservation technique resulting in a significant difference in the filling of the post-extraction socket with mineralized bone, respectively $94.7 \%$ in the study site and $63.3 \%$ in the control site [20]. Similar observations were made by Alzahrani et al. [29].

Some authorshave reported that the beneficial effect of PRF may be reduced, dependent on the technique of the procedure. According to Hauser et al. [30], mobilization of the flap may eliminate the beneficial effect of PRF on alveolar healing after extraction. In this case study, there was no possibility to perform a flapless surgical procedure. The flap was raised in order to have good access to necrotic tissue as, well as to close the whole bony defect with soft tissues. It is worth mentioning that PRF can be applied together with other biomaterials, such as decalcified freeze-dried bone graft (DFDBA) or calcium sulfate to maintain the width of the bone ridge after tooth extraction $[30,31,32]$. In the presented case, it was decided not to use any bone substitute because of the risk of complications with soft tissue healing and, as a result, increased risk of infection and sequestration of the bone graft.

Autologous platelet concentrates can be useful in the management of young, immature, necrotic, permanent teeth, to improve apical closure and preserve tooth vitality [33]. In this case study, the use of PRF probably had the potential to preserve pulp viability in the adjacent tooth 24 . Platelet-rich preparations can be also used for soft tissue augmentation as an alternative to connective tissue graft (CTG), usually in combination with a coronally advanced flap [34]. However, in the presented case, gingival augmentation was only partial. This could be explained by the fact that interproximal 
gingival augmentation is highly unpredictable, and that the patient was a heavy smoker; therefore, the healing potential was reduced.

The patient was informed during follow-up visits that CTG grafting is advisable in her case to improve soft tissue volume, but because of the treatment costs and the fact that she still smoked 10-15 cigarettes per day, no further mucogingival treatment was performed. Alternatively, the patient was advised to perform a prosthetic restoration using ceramic crowns in the left upper premolars to reconstruct the interproximal contact point and locate it more apically. This approach may result in partial regrowth of the interproximal soft tissue. Unfortunately, this treatment plan was not accepted by the patient.

Some authors have described allergic reactions after the use of paraformaldehyde-containing agents [5]. Kunisada et al. [5] showed a case of itchy urticarial erythema on a patient's skin, accompanied by systemic symptoms including wheezing, cough, dyspnea and dysphagia eight hours after application of a paraformaldehyde-containing root canal disinfectant. The authors removed the paraformaldehyde paste from the root of the treated toot and administered intravenous hydrocortisone, achieving clearance of symptoms within two days. In the presented case study, no systemic complications were present.

\section{CONCLUSIONS}

Dental practitioners should always be aware of possible complications after the use of devitalizing agents, and be prepared to diagnose and treat them properly. In the case where the use of a paraformaldehyde-containing paste is ncessary, it is important to follow the manufacturer's recommendations, and especially to secure the sealing of temporary restoration. For that reason, a glass-ionomer filling is the material of choice. Comprehensive history taking may help to avoid possible allergic reactions and risk of anaphylaxis to paraformaldehyde. A wide range of agents and methods of analgesia are available which enable proper pain management in most cases and painless endodontic treatment. For this reason, nowadays, the use of devitalizing pastes is limited. However, the paraformaldehyde-containing agents are still used and complications continue to be reported [1]. Based on the literature and the presented case study, it can be stated that the use of platelet-rich fibrin has the potential to reduce post-operative pain and promote tissue healing in patients treated for iatrogenic gingival and bone necrosis.

\section{REFERENCES}

1. Lee CH, Choi Y, Park S. Mandibular bone necrosis after use of paraformaldehyde paste. Restor Dent Endod. 2016; 41(4): 332-337. doi: $10.5395 /$ rde.2016.41.4.332

2. Jung RM, Rybak M, Milner P, Lewkowicz N. Local anesthetics and advances in their administration - an overview. J Pre Clin Clin Res. 2017; 11(1): 94-101. doi: https://doi.org/10.26444/jpccr/75153

3.https://baza-lekow.com.pl/devipasta/ (date of reviewing15.03.18).

4. Chen G, Sung PT. Gingival and localized alveolar bone necrosis related to the use of arsenic trioxide paste - Two case reports. J Formos Med Assoc. 2014; 113(3): 187-90. doi: 10.1016/j.jfma.2012.07.023

5. Kunisada M, Adachi A, Asano H, Horikawa T. Anaphylaxis due to formaldehyde released from root canal disinfectant. Contact Dermatitis. 2002; 47(4): 215-8. doi: 10.1034/j.1600-0536.2002.470405
6. Tortorici S, Burruano F, Difalco P. Maxillary bone necrosis following the use of formaldehyde containing paste: management and case series. Br Dent J. 2007; 203(9): 511-2. doi: 10.1038/bdj.2007.995

7. Ozgöz M, Yagiz H, Ciçek Y, Tezel A. Gingival necrosis following the use of a paraformaldehyde-containing paste: a case report. Int Endod J. 2004; 37(2): 157-61. doi: 10.1111/j.0143-2885.2004.00770

8. Athanassiadis B, George GA, Abbott PV, Wash LG. A review of the effects of formaldehyde release from endodontic materials. Int Endod J. 2014; 48(9). doi: 10.1111/iej.12389

9. Bilal M, M N Iqbal H. An insight into toxicity and human-health-related adverse consequences of cosmeceuticals - A review. Sci Total Environ. 2019; 670: 555-568. doi: 10.1016/j.scitotenv.2019.03.261

10. Pianigiani G, Betti C, Brunetti L. Response to: "Cytoplasmic dislocation of NPM1 and PU.1 in NPM1-mutated leukemia is obscured by paraformaldehyde fixation”. Br J Haematol. 2020; 189(3): 577-578. doi: $10.1111 /$ bjh.16544

11. Jang JH, Park SH, Jang HJ, Lee SG, Park JH, Jeong JW, Park CS. A Case of Recurrent Urticaria Due to Formaldehyde Release from RootCanal Disinfectant. Yonsei Med. 2017; 58(1): 252-254. doi: 10.3349/ ymj.2017.58.1.252

12. Syed M, Chopra R, Sachdev V. Allergic Reactions to Dental Materials-A Systematic Review. J Clin Diagn Res. 2015; 9(10): ZE04-9. doi: 10.7860/ JCDR/2015/15640.6589

13. Hülsmanna M, Hornecker E, Redeker M. Periodontal destruction and tooth loss following pulp devitalization with Toxavit: report of a case. Dent Traumatol. 1993; (9): 216-221. doi.org/10.1111/j.1600-9657.1993. tb00277

14. Elgendy EA, Shady TEA. Clinical and radiographic evaluation of nanocrystalline hydroxyapatite with or without platelet-rich fibrin membrane in the treatment of periodontal intrabony defects. J Indian Soc Periodontol. 2015; 19(1): 61-5. doi: 10.4103/0972-124X.148639

15. Dumlu A, Yalcinkaya S, Olgac V, Guvercin M. Osteomyelitis due to arsenic trioxide use for tooth devitalization. Int Endod J. 2007; 40(443): 317. doi.org/10.1111/j.0143-2885.2007.01230

16. Naik AR, Ramesh AV, Dwarkanath CD, et al. Use of autologous platelet rich plasma to treat gingival recession in esthetic periodontal surgery. J Indian Soc Periodontol. 2013; 17(3): 345-53. doi: 10.4103/0972124X.115665

17. Miron RJ, Pinto NR, Quirynen M, Ghanaati S. Standardization of relative Centrifugal Forces (RCF) in studies related to platelet rich fibrin. J Periodontol. 2019; 90,8: 817-820. doi: 10.1002/jper.18-0553

18. Naik B, Karunakar P, Jayadev M, Marshal VR. Role of Platelet rich fibrin in wound healing: A critical review. J Conserv Dent. 2013; 16(4): 284-93. doi: 10.4103/0972-0707.114344

19. Padma R, Shilpa A, Kumar PA, et al. A split mouth randomized controlled study to evaluate the adjunctive effect of platelet-rich fibrin to coronally advanced flap in Miller's class-I and II recession defects. J Indian Soc Periodontol. 2013; 17(5): 631-6. doi: 10.4103/0972-124X.119281

20. Malujda M, Gołowkin J, Droździk A. Use of platelet ricg fibrin (PRF) in socket preservation - current literature review and own experiences. Mag Stomatol. 2019; (1): 80-86.

21. Chmielewska M, Chmielewski K, Iwańczyk B, Wójtowicz A. Możliwość zastosowania PRP i PRF w sterowanej regeneracji tkanek w chirurgii stomatologicznej. Implants. 2014; 2: 12-16.

22. Kobayashi E, Flückiger L, Fujioka-Kobayashi M, et al. Comparative release of growth factors from PRP, PRF and advanced-PRF. Clin Oral Investing. 2016; 20(9): 2353-2360. doi: 10.1007/s00784-016-1719-1

23. Marty M, Noirrit-Esclassan E, Diemer F. Arsenic trioxide-induced osteo-necrosis treatment in a child: minireview and case report M. Eur Arch Paediatr Dent. 2016; (17): 419-422. doi: 10.1007/s40368-016-0250-Z

24. Yalc S, Aybar B, Haznedaroğlu F, Yücel E. Bilateral oroantral fistulas following devitalization of teeth by arsenic trioxide: a case report. J Endod. 2003; 29(3): 205. doi: 10.1097/00004770-20030300000010

25. Nowak J, Adamiec M, Iwańczyk B, Czerniuk M. Will the use of autologous blood derivatives be the gold standard in various medicine specialties in the 21st century? Hematol. 2020; 11(1): X-XX. doi: 10.5603/ Hem.2020.0002

26. Al-Hamed F, Tawfik M, Abdelfadil E. Clinical effects of platelet-rich fibrin (PRF) following surgical extraction of lower third molar. Saudi J Oral Dent Res. 2017; (8): 19-25. doi: 10.1016/j.sjdr.2016.05.002

27. Marenzi G, Riccitiello F, Tia M, et al. Influence of leukocyte- and platelet-rich fibrin (L-PRF) in the healing of simple postextraction sockets: a split-mouth study. BioMed Res Int. 2015; 2015: 369273. doi: $10.1155 / 2015 / 369273$

28. Temmerman A, Vandessel J, Castro A, et al. The use of leucocyte and platelet-rich fibrin in socket management and ridge preservation: a 
split-mouth, randomized, controlled trial. J Ciln Periodontol. 2016; 43(11): 990-999. doi: 10.1111/jcpe.12612

29. Alzahrani AA, Murriky A, Shafik S. Influence of platelet rich fibrin on post-extraction socket healing: A clinical and radiographic study. Saudi Dent J. 2017; 29(4): 149-155. doi: 10.1016/j.sdentj.2017.07.003

30. Hauser F, Gaydarov N, Badoud I, et al. Clinical and histological evaluation of postextraction platelet-rich fibrin socket filling: A prospective randomized controlled study. Implant Dent. 2013; 22(3): 295-303. doi: 10.1097/ID.0b013e3182906eb3

31. Thakkar DJ, Deshpande NC, Dave DH, et al. A comperative evaluation of extraction socket preservation with demineralized freeze-dried bone allograft alone and along with platelet-rich fibrin: A clinical and radigraphic study. Contemp Clin Dent. 2016; 7(3): 371-6. doi: 10.4103/0976-237X.188567

32. Kumar GN, Chaudhary R, Kumar I, et al. To assess the efficacy of socket plug technique using platelet rich fibrin with or without the use of bone substitute in alveolar ridge preservation: a prospective randomised controlled study. Oral Maxillofac Surg. 2018; 22(2): 135-142. doi: 10.1007/s10006-018-0680-3

33. Panda S, Mishra L, Arbildo-Vega HI, Lapinska B, Lukomska-Szymanska M, Khijmatgar S, Parolia A, Bucchi C, Fabbro MD. Effectiveness of Autologous Platelet Concentrates in Management of Young Immature Necrotic Permanent Teeth-A Systematic Review and Meta-Analysis. Cells. 2020; 9(10): 2241. doi: 10.3390/cells9102241

34. Panda S, Satpathy A, Chandra Das A, Kumar M, Mishra L, Gupta S, Srivastava G, Lukomska-Szymanska M, Taschieri S, Del Fabbro M. Additive Effect of Platelet Rich Fibrin with Coronally Advanced Flap Procedure in Root Coverage of Miller's Class I and II Recession Defects-A PRISMA Compliant Systematic Review and Meta-Analysis. Materials (Basel). 2020; 13(19): 4314. doi: 10.3390/ma13194314 